\title{
Erratum to: An avian-only Filippov model incorporating culling of both susceptible and infected birds in combating avian influenza
}

\author{
Nyuk Sian Chong', ${ }^{1,2}$ Benoit Dionne ${ }^{1}$. \\ Robert Smith? ${ }^{1,3}$
}

Published online: 15 April 2016

(C) Springer-Verlag Berlin Heidelberg 2016

\section{Erratum to: J. Math. Biol. DOI 10.1007/s00285-016-0971-y}

Unfortunately, in the original publication of the article, the first row of Table 2 was misplaced i.e. the entry $I_{b}<g_{3}$ should be the first row.

The corrected table is given below and the original article has been updated to reflect this change.

The online version of the original article can be found under doi:10.1007/s00285-016-0971-y.

$\bowtie$ Robert Smith?

rsmith43@uottawa.ca

Nyuk Sian Chong

nchon077@uottawa.ca

Benoit Dionne

bdionne@uottawa.ca

1 Department of Mathematics and Statistics, University of Ottawa, 585 King Edward Ave, Ottawa, ON K1N 6N5, Canada

2 School of Informatics and Applied Mathematics, Universiti Malaysia Terengganu, 21030 Kuala Terengganu, Malaysia

3 Faculty of Medicine, University of Ottawa, 451 Smyth Rd, Ottawa, ON K1H 8M5, Canada 
Table 2 Conclusions for Sects. 3-6

\begin{tabular}{|c|c|c|c|c|}
\hline & $S_{b}<h_{1}$ & $h_{1}<S_{b}<h_{2}$ & $h_{2}<S_{b}<h_{3}$ & $S_{b}>h_{3}$ \\
\hline$I_{b}<g_{3}$ & II & II & IV & II \\
\hline \multirow{3}{*}{$g_{3}<I_{b}<g_{2}$} & \multirow{3}{*}{ I } & $I_{b}<g_{8}: \mathrm{I}$ & $I_{b}<g_{7}<g_{6}<g_{4}$ : III & \multirow{3}{*}{ II } \\
\hline & & \multirow{2}{*}{$g_{8}<I_{b}: \mathrm{I}$} & $g_{7}<I_{b}<g_{6}<g_{4}:$ III & \\
\hline & & & $g_{6}<I_{b}:$ II & \\
\hline \multirow{3}{*}{$g_{2}<I_{b}<g_{1}$} & \multirow{3}{*}{ I } & $I_{b}<g_{8}<g_{4}: \mathrm{I}$ & \multirow{3}{*}{ I } & \multirow{3}{*}{ I } \\
\hline & & $g_{8}<I_{b}<g_{4}: \mathrm{I}$ & & \\
\hline & & $g_{8}<g_{4}<I_{b}: \mathrm{I}$ & & \\
\hline$g_{1}<I_{b}$ & $\mathrm{I}$ & I & $\mathrm{I}$ & I \\
\hline
\end{tabular}

